SECTION 2. Applied mathematics. Mathematical modeling.

Mahruy Saidalieva

PHD, Head of dept "Regulatorika" Centre for the development of software and hardware program complexes at Tashkent University of Informational Technologies,

Tashkent, Uzbekistan regulatorika@yahoo.com

Mohiniso Bahromovna Hidirova

PHD, Senior Scientist, Department of Regulatorika, Centre for the development of software and hardware program complexes at

Tashkent University of Informational Technologies,

Tashkent, Uzbekistan

regulatorika@yahoo.com

\title{
FUNCTIONAL-DIFFERENTIAL EQUATIONS OF BIOLOGICAL COMMUNITIES REGULATORIKA
}

Abstract: The functional-differential equations of biological community's number regulatorika are given. Quantitative functioning regularities for concrete cellular community of an organism immune system at AIDS are defined. dynamics.

Key words: mathematical model, functional-differential equations, chaos, nonlinear

\section{УДК 576.35:517.948}

\section{ФУНКЦИОНАЛЬНО-ДИФФЕРЕНЦИАЛЬНЫЕ УРАВНЕНИЯ РЕГУЛЯТОРИКИ БИОЛОГИЧЕСКИХ СООБЩЕСТВ}

Аннотация: Приведены функционально-дифференциальные у уравнения регуляторики численности биологических сообществ. Определены количественные закономерности функиионирования конкретного клеточного сообщества иммунной системы организма при СПИДе.

Ключевые слова: математическая модель, функционально-дифференциальные уравнения, хаос, нелинейная динамика.

При решении многих биологических задач требуется количественная оценка поведения биологических сообществ, объединенных выполнением некоторых общих функций, необходимых для жизнедеятельности системы, в рамках которой происходит существование рассматриваемого сообщества [1, с. 283-308; 2, с. 389; 3, с. 287-307; 4, с. 463-469; 5, с. 167-169; 6, с. 48-64; 7, с. 899-910]. Особенно это актуально для модельных исследований функиионирования регуляторных механизмов (регуляторики) клеточных сообществ организма [8, с. 41-46]. На основе принципов биологического эпиморфизма $[6$, c.50-51] и блочной структурно-функциональной организации живых систем [7, с.900] было разработано понятие функциональной единищь биологических сообществ (фебс) как связного множества (по пространству или (и ) по времени) с размножающимися (M), растущими $\left(\mathrm{B}_{1}\right)$, дифференцирующимися (D), выполняющими специфические функции $\left(\mathrm{S}_{1}, \mathrm{~S}_{2}, \ldots, \mathrm{S}_{\mathrm{n}} ; \mathrm{n}\right.$ - количество выполняемых специфических функций) и стареющими $\left(\mathrm{B}_{2}\right)$ элементами. В ходе функционирования системы «фебс», ее элементы последовательно переходят из одной однородной группы в соответствующую 
однородную группу элементов (рис.). Последнюю однородную группу $\left(\mathrm{B}_{2}\right)$ элементы покидают путем естественной гибели - апоптоза или (при необходимости) путем перехода на однородную группу дифференцирующихся элементов, где они дедифференцируются, готовясь к повторному выполнению специфических функций. Переходы элементов осуществляются с некоторыми временными затратами, что составляет временные взаимоотношения системы.

Для количественного описания изменения численностей элементов в конкретных группах фебс можно предложить, с учетом временных взаимоотношений, следующую систему функционально-дифференциальных уравнений регуляторики численности биологических сообществ [4, с. 463-469; 5, с. 167-169]

$$
\begin{aligned}
& \frac{d X_{1}(t)}{d t}=a_{1}\left(\prod_{k=1(k \neq 2,3)}^{n+3} X_{k}(t-1)\right) /\left(1+\sum_{j=1}^{n+4} \delta_{j} X_{j}(t)\right)+b_{1} X_{2}(t-1)-a_{2} X_{1}(t) \\
& \frac{d X_{2}(t)}{d t}=a_{2} X_{1}(t-1)+b_{2} X_{3}(t-1)-\left(b_{1}+a_{3}\right) X_{2}(t) \\
& \frac{d X_{3}(t)}{d t}=a_{3} X_{2}(t-1)+b_{3} X_{n+4}(t-1)-\left(b_{2}+\sum_{k=4}^{n+3} a_{k}\right) X_{3}(t) \\
& \frac{d X_{k}(t)}{d t}=a_{k} X_{3}(t-1)-a_{n+4} X_{k}(t) ; \quad k=4,5, \ldots, n+3 ; \\
& \frac{d X_{n+4}(t)}{d t}=a_{n+4} \sum_{k=4}^{n+3} X_{k}(t-1)-\left(b_{3}+c\right) X_{n+4}(t)
\end{aligned}
$$

где $a_{1}$ - постоянная скорости размножения элементов в $\mathrm{M} ; a_{\mathrm{i}},(i=2, \ldots, n+4), b_{\mathrm{j}}(j=1,2,3)$ - постоянные скорости прямых и обратных переходов; $\delta_{k}(k=1,2, \ldots, 6)$ - параметры репрессии активности элементов в $\mathrm{M} ; c$ - постоянная скорости естественной гибели апоптоза стареющих элементов.

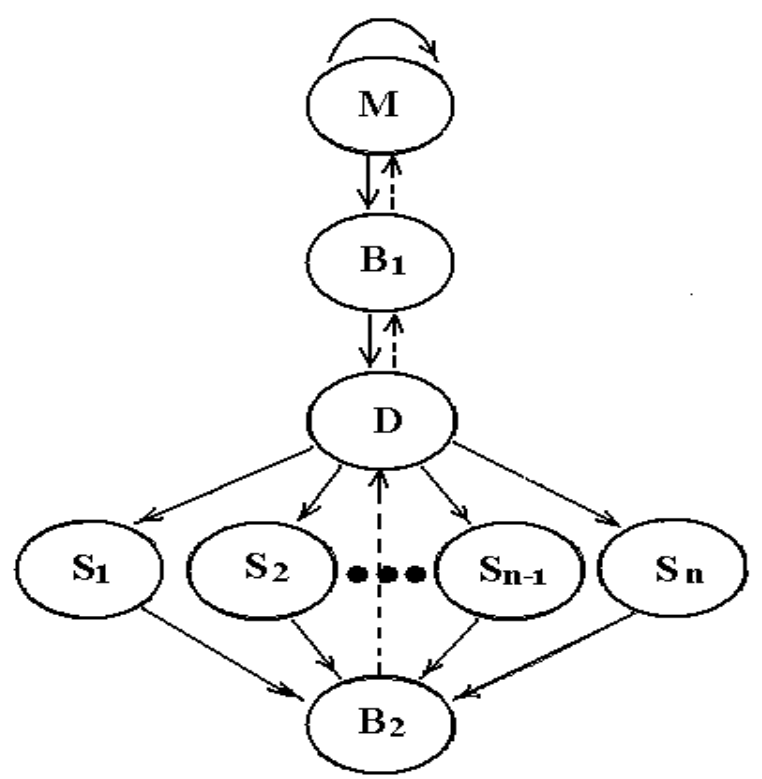

Рисунок 1 - Схема клеточных переходов в фебс.

Уравнения регуляторики динамики численности биологических сообществ (1) составляют замкнутую систему функционально-дифференциальных уравнений. Теоремы существования и единственности решений, а также приближенные решения (1) на РС могут быть получены с использованием метода последовательного 
интегрирования Беллмана-Кука при задании начальной функции на отрезке единичной длины.

Формулирование понятия функциональной единицы биологических сообществ «фебс», разработка соответствующих модельных и программных обеспечений позволили количественно анализировать регуляторные механизмы функциональной единицы клеточных сообществ животных и растительных организмов в норме и при аномалиях. На основе качественного и количественного анализа конкретных видов функционально-дифференциальных уравнений (1) и их модельных систем выявлены закономерности динамики численности популяций в последовательных зонах «фебс» (деления, роста, дифференцировки, выполнения специфических функций и старения), а путем имитационного моделирования определены количественные закономерности функционирования конкретных клеточных структур с учетом пространственной архитектоники клеток и регуляции внутриклеточных процессов.

Рассмотрим некоторые вопросы математического и компьютерного моделирования регуляторики динамики конкретного клеточного сообщества иммунной системы организма при ВИЧ \СПИД с использованием уравнений (1). ВИЧ инфекция, поражая СД4 лимфоциты иммунной системы, в конечном итоге приводит к резкому ослаблению иммунитета (СПИД) и гибели организма $[9$, c.3]. Для анализа регуляторики динамики численности СД4 лимфоцитов мы упростили (1), рассматривая только одну группу клеток $\mathrm{S}$, выполняющих специфическую функцию (СД4 лимфоциты). Поскольку нас интересуют наиболее общие механизмы поддержания гомеостазиса СД4 лимфоцитов в течение жизни организма, мы предполагаем, что в группах $\mathrm{B}_{1}, \mathrm{D}, \mathrm{S}$ и $\mathrm{B}_{2}$ изменение численностей клеток происходит быстрее, чем в группе M, и обычно транслирует его. Следовательно можно допустить равновесный характер изменения численности клеток в зонах $\mathrm{B}_{1}, \mathrm{D}, \mathrm{S}$ и $\mathrm{B}_{2}$ и систему (1) упростить до одного уравнения

$$
\frac{\theta}{h} \frac{d X(t)}{d t}=\rho X^{2}(t-1) e^{-X(t-1)}-X(t),
$$

где $X(t)$ - функция, выражающая численность пролиферативных клеток тимуса, воспроизводящих СД4 лимфоциты; $\theta$ - среднее время их жизни; $h$ - интервал времени, необходимого для осуществления обратной связи в иммунной системе организма; $\rho$ - параметр вирусной нагрузки, выражающий скорость деления клеток в пролиферативном пуле иммунной системы.

Предположим, что среднее время деления пролиферативных клеток тимуса намного меньше интервала времени, необходимого для осуществления обратной связи в иммунной системе организма, т.е. $\theta<<$. Тогда, для анализа динамики численности СД4 лимфоцитов могут быть использованы модельные системы (2) в виде функционального уравнения

$$
X(t)=\rho X^{2}(t-1) e^{-X(t-1)}
$$

и его дискретного аналога

$$
X_{k+1}=\rho X_{k}^{2} e^{-X_{k}}, k=0,1, \ldots,
$$

где $X_{k}$ - величина, выражающая численность пролиферативных клеток иммунной системы на $k$-ом шаге жизнедеятельности организма. Решение (4) можно визуально оценивать с помощью построения диаграмм Ламерея и вычисления энтропии Колмогорова и показателя Ляпунова на РС.

Результаты анализа поведений решений (4) показывают возможность существования режимов покоя, стационарного состояния, автоколебаний, нерегулярных колебаний и эффекта срыва решений к тривиальному аттрактору - 
"черной дыре". Обычно, нерегулярные колебания и "черная дыра" идентифицируются аномалиями биосистем [10, с. $164 ; 11$, с.413; 12, с.107].

Закономерности возникновения и развития нерегулярных колебаний и "черной дыры" были исследованы с помощью (4) путем анализа динамики значений показателя Ляпунова и построения диаграмм Ламерея (при различных значениях параметра $\rho$ уравнения (4)) на РС с использованием специальной программы “SW-FDE$3 "[13$, c.6].

Таким образом, последовательное увеличение параметра вирусной нагрузки, наблюдающееся при ВИЧ инфекции, приводит к переходу в режим нерегулярных колебаний и, в дальнейшем, к резкому деструктивному уменьшению воспроизводства СД4 лимфоцитов. В области нерегулярных колебаний численность популяции СД4 лимфоцитов ведет себя непредсказуемым образом, а в области «черной дыры» происходит остановка деления пролиферативных клеток тимуса, воспроизводящих СД4 лимфоциты. В случае нахождения популяции СД4 лимфоцитов в области аномалий возникает вопрос об уводе ее в область автоколебаний и (или) в область стационарного режима. Результаты количественных исследований (4) на РС показали существование в области нерегулярных колебаний малых регионов регулярного поведения (rwindows) рассматриваемой системы. Наличие r-windows позволяет временно разрешить проблему вводом системы в ближайший r-window, чтобы затем вывести систему из области нерегулярных колебаний. Следовательно, эффективным является организация маршрута увода системы из области нерегулярности по цепочке, состоящей из rwindows. Быстротечность деструктивных изменений в случае эффекта «черной дыры» усложняет управление поведением популяции СД4 лимфоцитов. Здесь требуется оценка времени нахождения системы в бассейне функционального аттрактора и разработка эффективных мер по переброске системы в зону детерминированного хаоса, а затем в зону регулярных колебаний. Следует отметить, что управление должно быть «щадящим» с минимальным уровнем нагрузки. В области аномалий естественным является принятие за величину нагрузки уровень нерегулярности состояния $(H)$ системы. Величина «Н» может быть вычислена на основе значений энтропии Колмогорова или показателя Ляпунова.

Результаты модельных исследований показывают, что хронический рост значения параметра вирусной нагрузки приводит к аномальному поведению численности СД4 лимфоцитов. Нарушается стационарное состояние, возникают автоколебания с переходом к нерегулярным колебаниям, а далее к эффекту "черной дыры" - резкому уменьшению численности СД4 лимфоцитов и развитию СПИД. Следует отметить, что здесь для объяснения СПИД не требуется привлечения концепции “предела Хейфлика". Из свойства общности модельных исследований следует, что такая же картина должна наблюдаться при любых аномалиях иммунной системы с хроническим ростом величины вирусной нагрузки.

\section{References:}

1. Bocharov G.A. Modelling the dynamics of LCMV infection in mice: conventional and exhaustive CTL responses // J. Theor. Biol., 1998. V.192, No 3. - pp.283-308.

2. Karr J.R., Sanghvi J.C., Macklin D.N., Gutschow M.V., Jacobs J.M., Bolival Jr. B., Assad-Garcia N., Glass J.I., Covert M.W. A whole-cell computational model predicts phenotype from genotype// Cell. 2012. - vol. 150. - pp. 389-401.

3. Акбердин И.Р., Казанцев Ф.В., Ермак Т.В., Тимонов В.С., Хлебодарова Т.М., Лихошвай В.А.«Электронная клетка»: проблемы и перспективы // Математическая биология и биоинформатика. 2013. Т. 8. No 1. с. 287-307. 
4. Saidalieva M. Modelling of Regulation Mechanisms of Cellular Communities //Scientiae Mathematicae Japanicae, 2003. Vol. 58. No 2. - pp. 463-469.

5. Saidalieva M. Mathematical modeling of regulation mechanisms of cellular communities // International Scientific and Practical Conference «Innovation-2001». Tashkent, 2001. - pp. 167-169.

6. Рашевски Н. Модели и математические принципы в биологии //Теоретическая и математическая биология М.: Мир, 1968, с. 48-64

7. Серавин Л.Н. Происхождение эукариотной клетки. IV. Общая гипотеза аутогенного происхождения эукариот //Цитология. 1986. Т. 28, N 9, с. 899-910

8. Хидиров Б.Н. Об одном методе исследования регуляторики живых систем //Вопросы кибернетики. - Ташкент, 1984. - Вып. 128., с. 41-46.

9. Perelson A.S., Nelson P.W. Mathematical Analysis of HIV-1 Dynamics in vivo // SIAM Review, 1999. V. 41. No 1. - pp. 3-44.

10. Saidalieva M. Mathematical and Computer Modelling Regulatorika of Organisms Cellular Communities at Anomalies //Scientiae Mathematicae Japonicae, 2008. Vol. 67. No 2. pp. 161-171.

11. Hidirov B.N. Modelling of regulation mechanisms of living system // Scientiae Mathematicae Japonicae, 2003.Vol. 58, No 2. - pp. 407-413.

12. Гласс Л., Мэки М. От часов к хаосу. Ритмы жизни. М.: Мир, 1991. - с. 107.

13. Хидиров Б.Н., Хидирова М.Б. Программа качественного анализа функциональнодифференциальных уравнений “SW-FDE-3”. Патентное ведомство РУз, 2004. Свидетельство № DGU 00720. - с. 6. 